\title{
Atrial Excitation Sequence during Ectopic Atrial Beats in the Rabbit
}

\author{
Atsushi Iто, M.D.
}

\begin{abstract}
SUMMARY
The propagation process of the atrial excitation was investigated during ectopic atrial rhythms evoked by electrical pacing through stimulation at several sites in 35 isolated rabbit atria using intracellular microelectrodes. Excitation sequence was correlated with the atrial deflections in a distant bipolar electrogram representing a cranio-caudal atrial activation. The conduction velocity was faster along the crista terminalis but slower across the coronary sinus ostium than in the ordinary atrial muscle. These differences in conduction velocity resulted in the upward atrial deflection on the distant bipolar electrogram in the rhythm which was produced by stimulation at the right side of the lower interatrial septum. On stimulation of the left atrial appendage, Bachmann's bundle displayed a function as a preferential conduction pathway in the case of left to right direction of excitation, as in left atrial rhythm. On stimulation of the postero-inferior region of the left atrium, the region around the coronary sinus ostium was the first area in the right atrium to be activated. Inversely, the impulse originating from the coronary sinus ostium was conducted relatively fast to the postero-inferior region of the left atrium. These findings suggested that there might be a preferential conduction pathway along the coronary sinus vein or its neighbouring tissues.
\end{abstract}

\section{Additional Indexing Words:}

Intracellular microelectrodes Preferential pathways Bachmann's bundle A distant bipolar electrogram Coronary sinus orifice Atrioventricular conduction

I clinical electrocardiography, the diagnosis of supraventricular arrhythmias is based on the polarity and morphology of the $\mathbf{P}$ wave and the duration of the P-R or R-P interval. Diagnostic criteria are based on the hypothesis proposed by Lewis ${ }^{1 /}$ and others. ${ }^{2)}$ Lewis hypothesized that the spread of activation in the atria was in a radial manner at a uniform conduction velocity from its site of origin and that it might be likened to the spread of fluid poured upon a flat surface, its edge advancing in an ever widening circle. According to this hypothesis, atrial excitation originating from a site near the $\mathrm{A}-\mathrm{V}$ node should result in negative $P$ waves in leads II, III, and aVF. However, it has

From the Research Institute of Environmental Medicine, Nagoya University, Furo-cho, Chikusaku, Nagoya, Japan.

Received for publication March 28, 1974. 
been reported that it is not necessarily true. ${ }^{3 /, 4)}$ Moore et $\mathrm{al}^{3)}$ concluded that activation of the canine atria originating from the region of the coronary sinus or A-V node did not result in inversion of the $\mathrm{P}$ wave in electrocardiographic leads II, III, and aVF unless there was concomitant damage to atrial myocardium. They suggested the participation of specialized atrial tracts in determining the polarity, morphology and duration of ectopic $\mathrm{P}$ waves. In sinus rhythm, in fact, there have been many investigators supporting the conception that there are specialized conduction tracts or preferential pathways in the atria. ${ }^{5)-12)}$ However, in the past, there have been few reports which showed the spread of atrial excitation in supraventricular arrhythmias. In the present study, atrial excitation sequence was mapped, to clarify the relation between the excitation process and the morphology of $\mathrm{P}$ waves during ectopic atrial beats.

\section{METHOD}

Thirty-five rabbits, weighing $2.2-3.5 \mathrm{Kg}$, were used in the present study. Each rabbit was anesthetized by intravenous administration of $30-40 \mathrm{mg}$ of sodium pentobarbital per $\mathrm{Kg}$ of body weight. The chest was opened and the heart was removed as quickly as possible. The isolated heart was constantly perfused through the

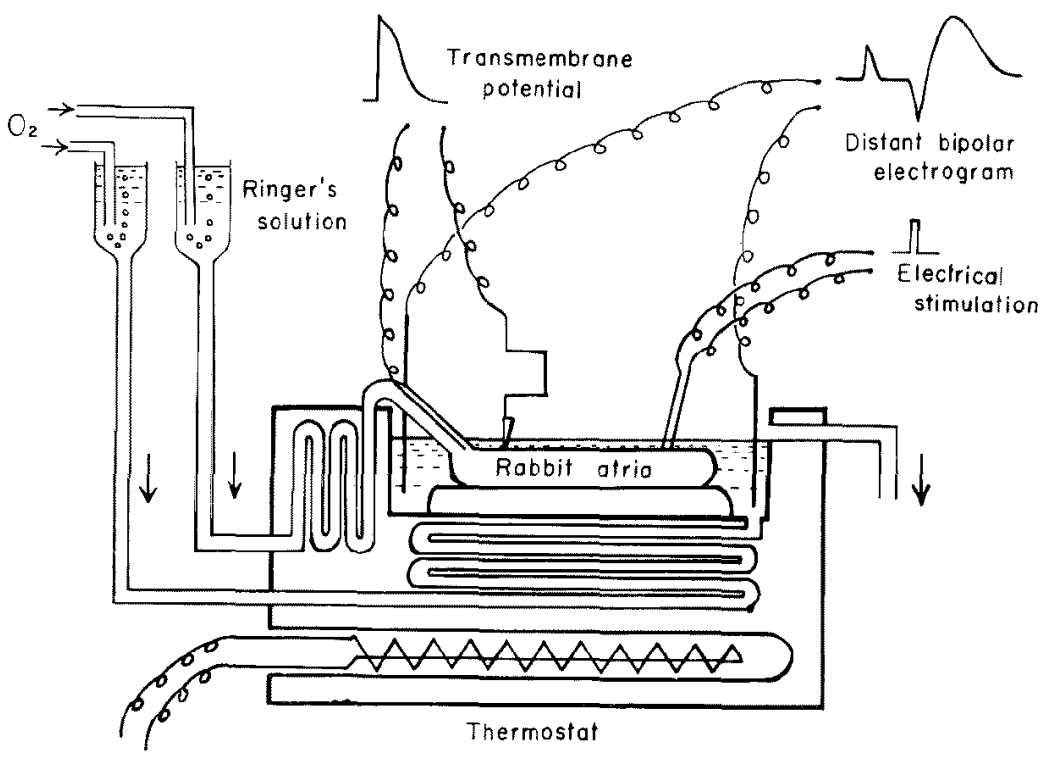

Fig. 1. An outline of the experimental apparatus. Transmembrane action potentials, a distant bipolar lead electrogram and a close bipolar lead electrogram (not shown) were recorded during perfusion of rabbit atria in the tissue bath by means of Langendorff's method. 
aorta with fully oxygenated Ringer's solution at $34-37^{\circ} \mathrm{C}$ by means of Langendorff's method. The free walls of the right and left ventricles were excised and the right atrium was opened by incising its appendage in a cranio-caudal direction with care not to damage the crista terminalis. Openings of artery branches which were transected during removal of ventricular free walls were ligated to control bleeding and maintain coronary flow through the specimen. After the specimen was fastened to a supporting frame with the exposed right atrial endocardial surface facing upward, it was placed in a tissue bath perfused with fully oxygenated Ringer's solution at a constant temperature of $34-37^{\circ} \mathrm{C}$. Figs. 1 and 2 show the experimental set up and the schematic illustration of the right atrial endocardial surface respectively.

Experiments were performed during artificial electrical pacing at a constant frequency of about $110 / \mathrm{min}$. The impulses for stimulation were rectangular pulses, which were 2 times threshold intensity and $3 \mathrm{msec}$ in duration. Abnormal atrial activation sequences were induced by pacing the specimen through stimulation at the sites shown in Fig. 3. These sites were as follows: sinus node region, the site near the coronary sinus ostium on the right side of the lower interatrial septum (LIS), the site lateral to the coronary sinus ostium on the extended region of the right segment of the crista terminalis (RCT), the posterior portion of the left atrial appendage (LAA), the postero-inferior region of the left atrium (PLA), the middle

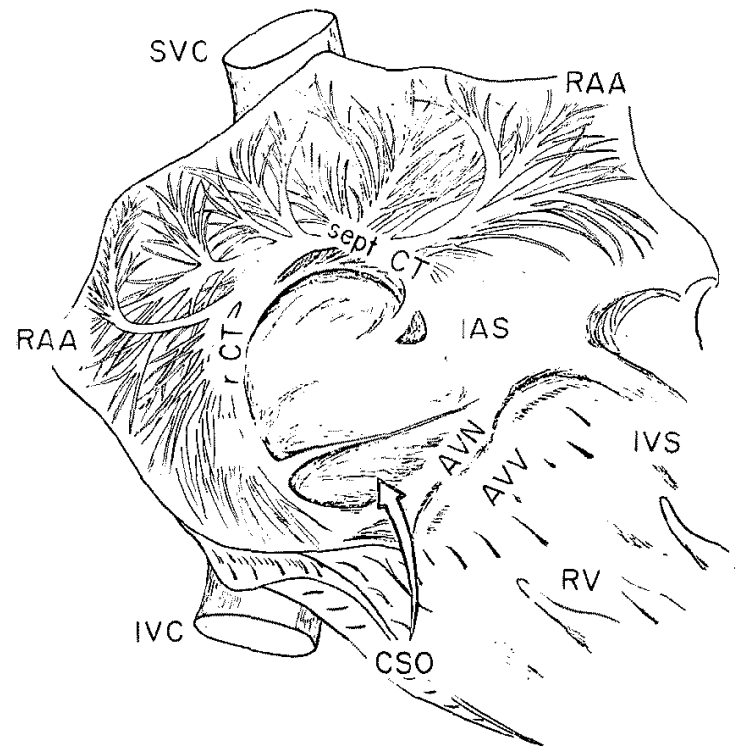

Fig. 2. Schematic illustration of the right atrial endocardial surface where microelectrodes impaled. The electrodes for the distant bipolar electrogram were placed in the bath on the extension of the line connecting the centers of the superior vena cava orifice and the coronary sinus orifice. SVC: superior vena cava, CSO: coronary sinus ostium, IVC: inferior vena cava, AVN: atrioventricular node, AVV: atrioventricular valves, IAS: interatrial septum, IVS: interventricular septum, RAA: right atrial appendage, sept GT: the septal segment of the crista terminalis, r GT: the right segment of the crista terminalis, RV: right ventricle. 


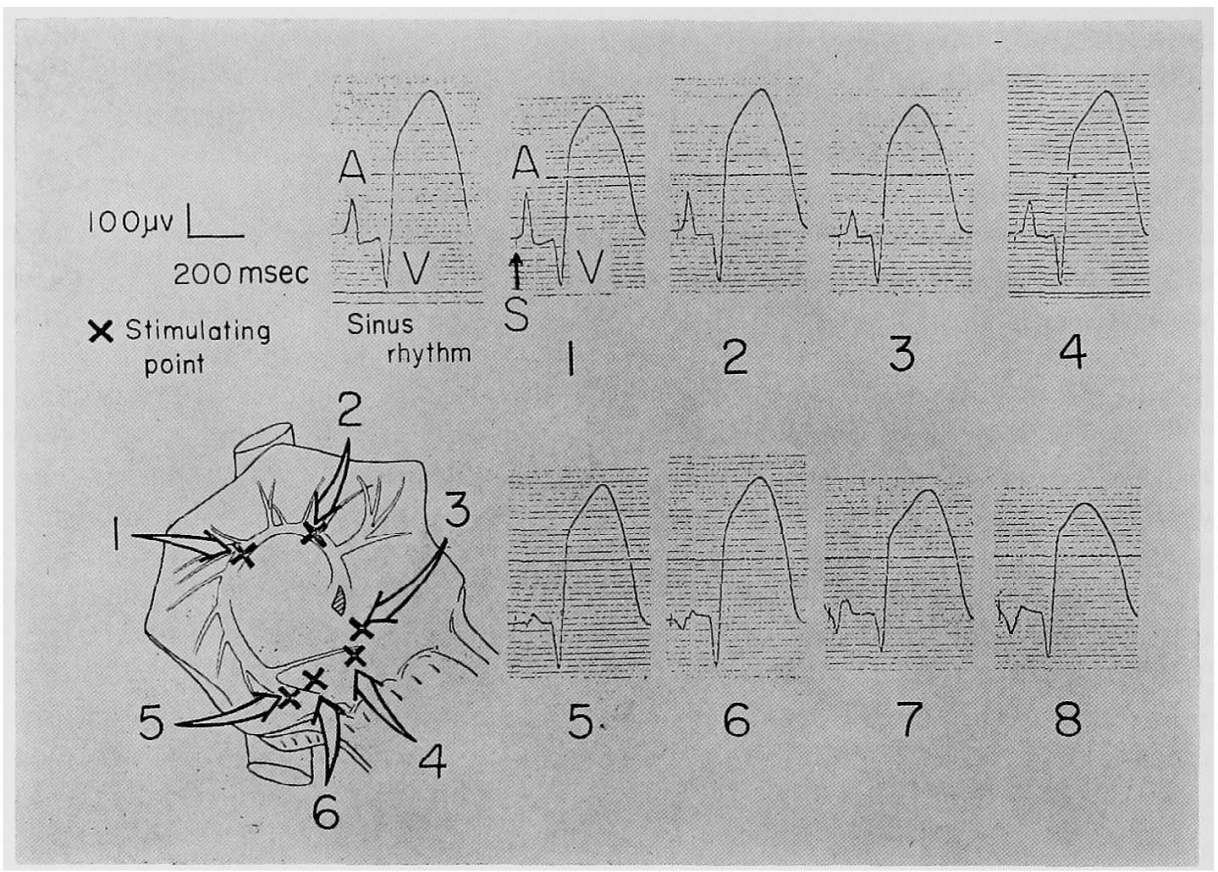

Fig. 3. Examples of the distant bipolar electrograms recorded from one specimen during pacing from each of 6 right atrial sites shown on the left, and 2 left atrial sites not illustrated. In each record, an initial small spike (S) is a stimulus artifact. The second small deflection (A) is an atrial deflection. The large downward and sharp deflection followed by a slow and upward convexed deflection (V) indicates a ventricular activation. Each number below the electrograms indicates the stimulating point. I: SA nodal region, 2: middle region of the septal segment of the crista terminalis, 3: LIS, 4: sept CSO, 5: RGT, 6: lat GSO, 7: LAA, 8: PLA.

part in the coronary sinus ostium (mid. CSO), the septal side in the coronary sinus ostium (sept. CSO), the lateral side in the coronary sinus ostium (lat. CSO).

In each experiment, transmembrane action potentials were obtained from 36 to 68 points of the right atrial endocardium including $\mathrm{A}-\mathrm{V}$ node and bundle of His. The vernier scale attached to the micromanipulator was used to position the points where microelectrodes impaled. The arrival time of the activation wave at points penetrated by intracellular microelectrodes was determined by measuring the interval from the artificial spike of the stimulating impulse to the onset of the sharp depolarization of the action potentials in each record. By mapping out the arrival time on the sketch of the atrium, the sequence of excitation in the right atrium was determined.

Simultaneously with recording of the transmembrane action potentials, close bipolar electrograms and a distant bipolar electrogram were recorded. The close bipolar plunge electrodes were used to record the activation times at given points on the right and left atria. These activation times were used to estimate changes in the specimen during the elapsed time in the experiment. The distant bipolar electrogram was used to record atrial activation in a cranio-caudal direction, and 
so the electrodes for it were located in the periphery of the tissue bath on the extension of the line connecting the center of the superior vena caval orifice and the coronary sinus orifice. Since the right atrium was opened by incising its appendage in a cranio-caudal direction, it was thought that the incision would not much affect the polarity of the atrial deflection in the distant bipolar electrogram and that the distant bipolar electrogram would represent a cranio-caudal atrial activation. The electrogram displayed an upward deflection when the potential of the electrode on the side of the coronary sinus orifice was relatively positive to the potential of the other electrode. The atrio-ventricular conduction time was determined from measurement of the interval from the onset of the atrial deflection to the onset of the ventricular deflection on the distant bipolar electrogram, and corresponded to the P-R interval of the conventional electrocardiogram. The electrodes used for the distant bipolar lead, the close bipolar leads and electrical pacing were made of fine silver wire insulated excepting for the tip ends, and the interpolar distance of the electrodes for the close bipolar leads and electrical pacing was about $1 \mathrm{~mm}$. Intracellular microelectrodes were made of pulled glass-capillary tubes filled with 3 mol $\mathrm{KCl}$ and with a tip-resistance of $10-30 \mathrm{M} \Omega$.

The transmembrane action potentials were amplified through a preamplifier (Nihon Koden Co, MZ-3B) and a D-C amplifier (Yokogawa Electric Works, Type3125). The output from the preamplifier was also introduced to a cathode ray oscilloscope (Nihon Koden Co, VG-6) for monitoring the transmembrane potentials. The close bipolar electrograms were amplified through a biophysical ME amplifier (Yokogawa Electric Works, BE-A 11). The distant bipolar electrogram was amplified through a Fukuda Denshi Co, UA-210 biophysical amplifier. These curves were simultaneously recorded by a photographic recorder (Yokogawa Electric Works, EMO-62) at a paper speed of $10 \mathrm{~cm} / \mathrm{sec}$.

For the convenience of describing the experimental results in this paper, the crista terminalis was divided into 2 parts separated at a point near the SA node. The part running along the atrial free wall was termed as the right segment and the other part extending into the atrial septum was termed as the septal segment according to Yamada et al. ${ }^{9}$

\section{Results}

The Polarity of the atrial deflections and the atrio-ventricular conduction time in the distant bipolar electrograms

The polarity of the atrial deflections and the atrio-ventricular conduction time were investigated in 13 experiments. In these experiments, the distant bipolar electrograms were recorded during pacing from 5 to 8 different sites in the same specimen. In Fig. 3, a representative case is shown. In each distant bipolar electrogram, a small mono- or di-phasic deflection (A) indicates an atrial activation and a large, downward and sharp deflection followed by a slow and upward convexed deflection (V) indicates a ventricular activation. A small spike preceding the atrial deflection (S) is a stimulus artifact.

When the specimen was paced from the $\mathrm{S}-\mathrm{A}$ nodal region (point 1 in 
Fig. 3), the atrial deflections almost always were positive and nearly the same as those in spontaneous sinus rhythm. The polarity of the atrial deflections changed little even when the stimulating point was moved along the septal segment of the crista terminalis from the region near the SA node to the region of the lower interatrial septum (LIS: point 3 in Fig. 3), except that the positive deflection was slightly preceded by a negative deflection as the stimulating point approached the coronary sinus ostium or the AV nodal region. In the case shown in Fig. 3, the atrial deflections were positive (point 2 and 3 in Fig. 3).

When the specimen was paced from the site lateral to the coronary sinus ostium on the extended region of the right segment of the crista terminalis (RCT: point 5 in Fig. 3), the polarity of the atrial deflection was diphasic (negative-positive) or isoelectric.

When the specimen was paced from the middle part or lateral side in the coronary sinus ostium (mid. CSO or lat. CSO: point 6 in Fig. 3), the atrial deflection was predominantly negative in most cases.

When the specimen was paced from the septal side in the coronary sinus ostium (sept. GSO: point 4 in Fig. 3), the polarity of the atrial deflection was intermediate between that in pacing from mid. CSO and that in pacing from LIS. It was diphasic (negative-positive).

When the specimen was paced from the posterior portion of the left atrial appendage (LAA: point 7 in Fig. 3), the polarity of the atrial deflection was diphasic (negative-positive).

When the specimen was paced from the postero-inferior region of the left atrium (PLA: point 8 in Fig. 3), the polarity of the atrial deflection was predominantly negative and it was similar to that during pacing from mid. CSO.

For convenience, the atrio-ventricular conduction time in each ectopic rhythm is shown as a percentage of that during pacing from the SA node. That is, the A-V conduction time during pacing from the SA nodal region is expressed as 100: 80.2 $\pm 11.0(\mathrm{~N}=10)$ during pacing from LIS, $91.4 \pm 7.0$ $(\mathrm{N}=6)$ during pacing from $\mathrm{RCT}, 92.9 \pm 7.7(\mathrm{~N}=10)$ during pacing from mid. to lat. $\mathrm{CSO}, 80.6 \pm 6.8(\mathrm{~N}=11)$ during pacing from sept. CSO, $103.6 \pm 19.5$ $(\mathrm{N}=7)$ during pacing from LAA, $103 \pm 15.5(\mathrm{~N}=3)$ during pacing from PLA. It was significantly shortened during pacing from the lower interatrial septum and from the coronary sinus ostium.

\section{The spread of the right atrial activation}

When the specimen was paced from the SA nodal region (Fig. 4), the impulse descended through both the right and the septal segments of the crista terminalis and activated the atrial side of the AV node in about $40 \mathrm{msec}$. 


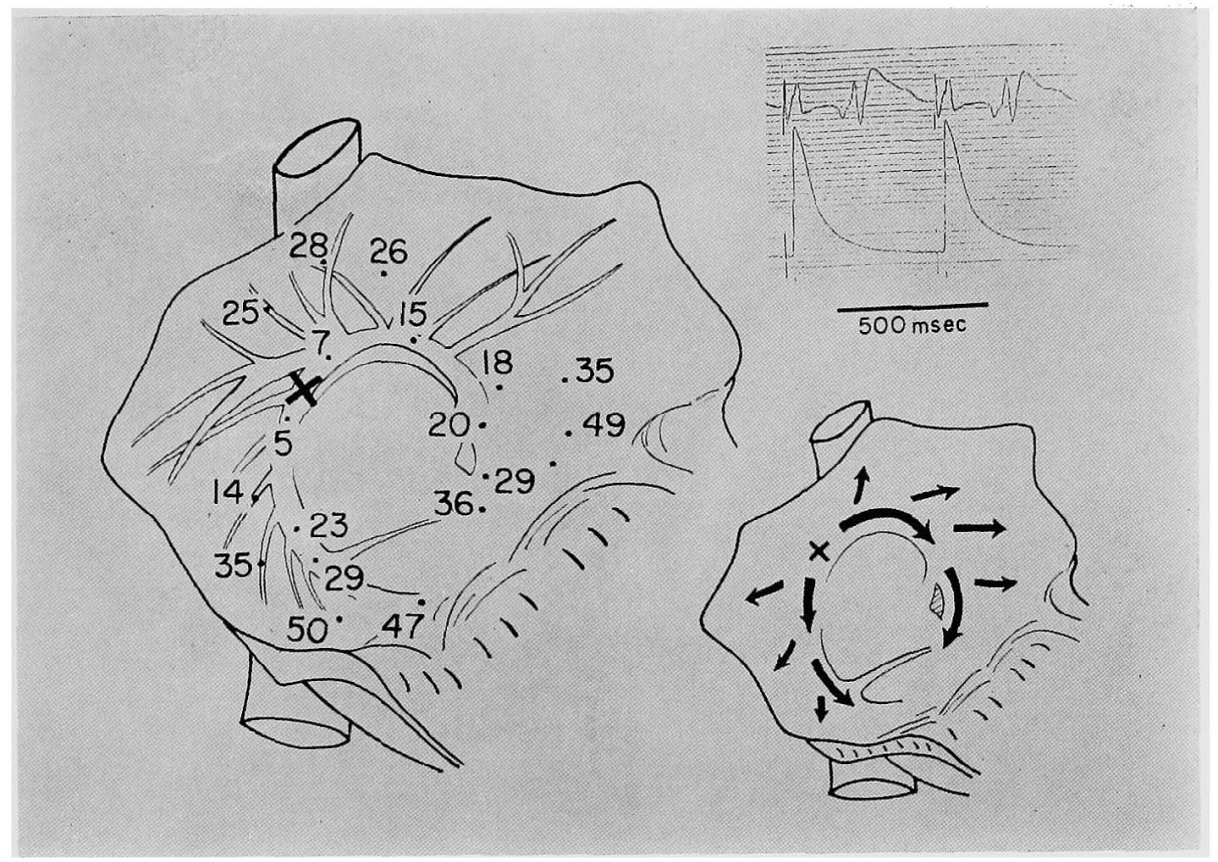

Fig. 4. Activation time of the right atrial endocardium during pacing from the SA nodal region. Left: Dots indicate the points where microelectrodes impaled. Numbers are activation times in msec. Right bottom: Schematic illustration of the spread of the right atrial activation summarized from 3 experiments. Thick arrows indicate fast conduction and thin arrows slow conduction.

The conduction velocity along each segment of the crista terminalis was about $100 \mathrm{~cm} / \mathrm{sec}$. The pattern of the right atrial activation during stimulation at the SA nodal region was similar to that which was shown in past reports.

When the specimen was paced from the right side of the lower interatrial septum (LIS, Fig. 5), the activation wave was transmitted faster upward along the septal segment of the crista terminalis and rather slowly across the coronary sinus ostium. The activation wave, which was conducted upward along the septal segment of the crista terminalis, spread around the anterior edge of the superior vena cava orifice and arrived near the SA nodal region. And then it descended along the right segment of the crista terminalis. On the other hand, the activation wave which was conducted across the coronary sinus ostium arrived at the opposite ridge of the ostium. These 2 activation waves collided with each other at the site lateral to the intercaval region on the right segment of the crista terminalis in $55 \mathrm{msec}$.

The approximate conduction velocity was $40-100 \mathrm{~cm} / \mathrm{sec}$ along the crista terminalis, $50 \mathrm{~cm} / \mathrm{sec}$ through the ordinary atrial muscle and $25 \mathrm{~cm} / \mathrm{sec}$ across 


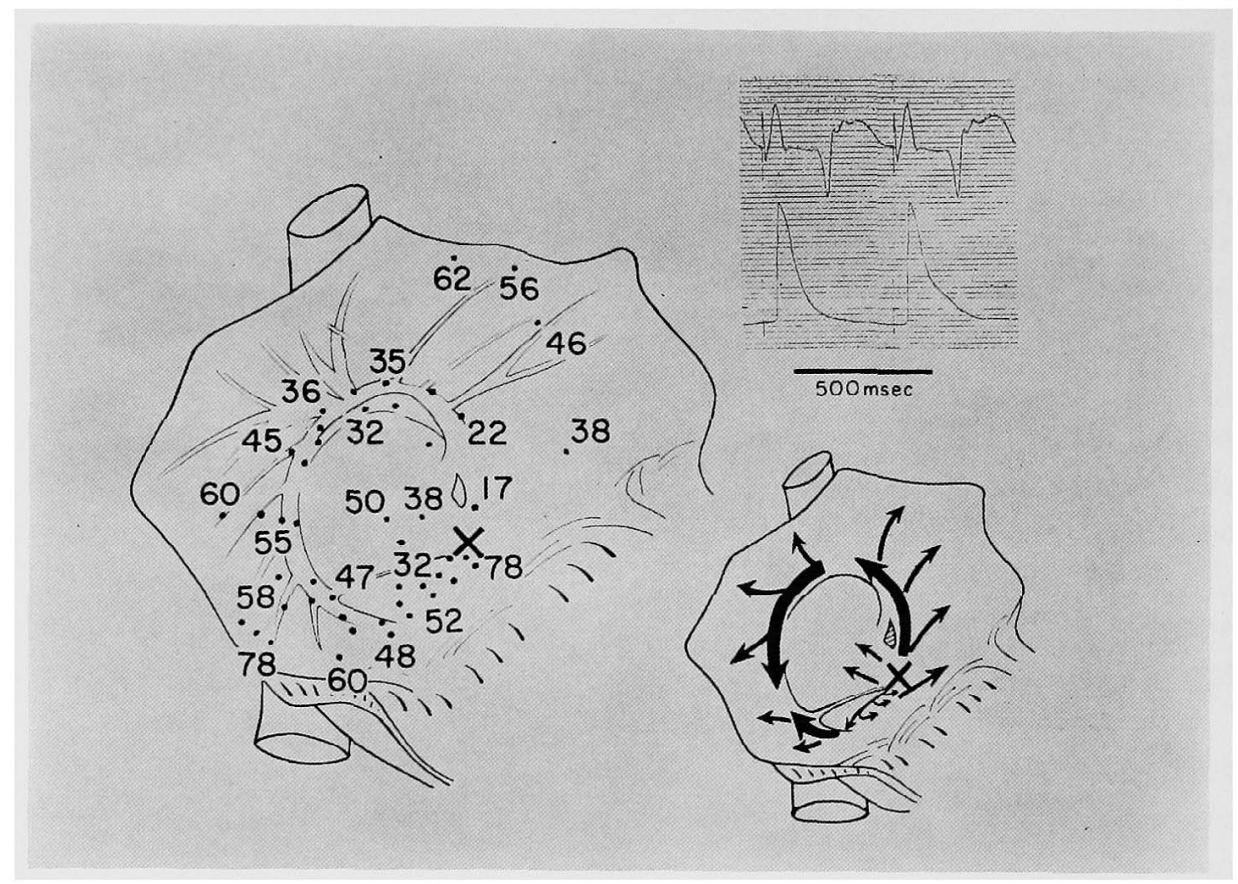

Fig. 5. Activation time of the right atrial endocardium during pacing from LIS. Demonstration of the panels is the same as in Fig. 4.

the coronary sinus ostium. These differences in conduction velocity contributed in the determination of the right atrial activation process. There are relatively wide variations in the conduction velocity along the crista terminalis probably according to whether the activation wave spread in parallel to thick muscle bundles or not. In the total excitation process, however, maximal conduction velocity along the crista terminalis was $80-100 \mathrm{~cm} / \mathrm{sec}$. There was no apparent difference in the conduction velocity between antegrade and retrograde conduction. As shown in Fig. 5, only a small portion of the right atrium was activated before the impulse arrived near the SA nodal region at about $35 \mathrm{msec}$. Most of the right atrial muscles were activated by the impulse propagated from the crista terminalis and a relatively large part of the activation wave in the ordinary atrial muscle was conducted in a craniocaudal direction rather than a caudo-cranial direction.

When the specimen was paced from RCT (Fig. 6), the activation wave was transmitted rapidly along the right segment of the crista terminalis and slowly across the coronary sinus ostium. The activation wave, which was transmitted upward along the right segment of the crista terminalis, was then transmitted toward the interatrial septum around the anterior edge of the superior vena cava orifice, and then downward along the septal segment of the 


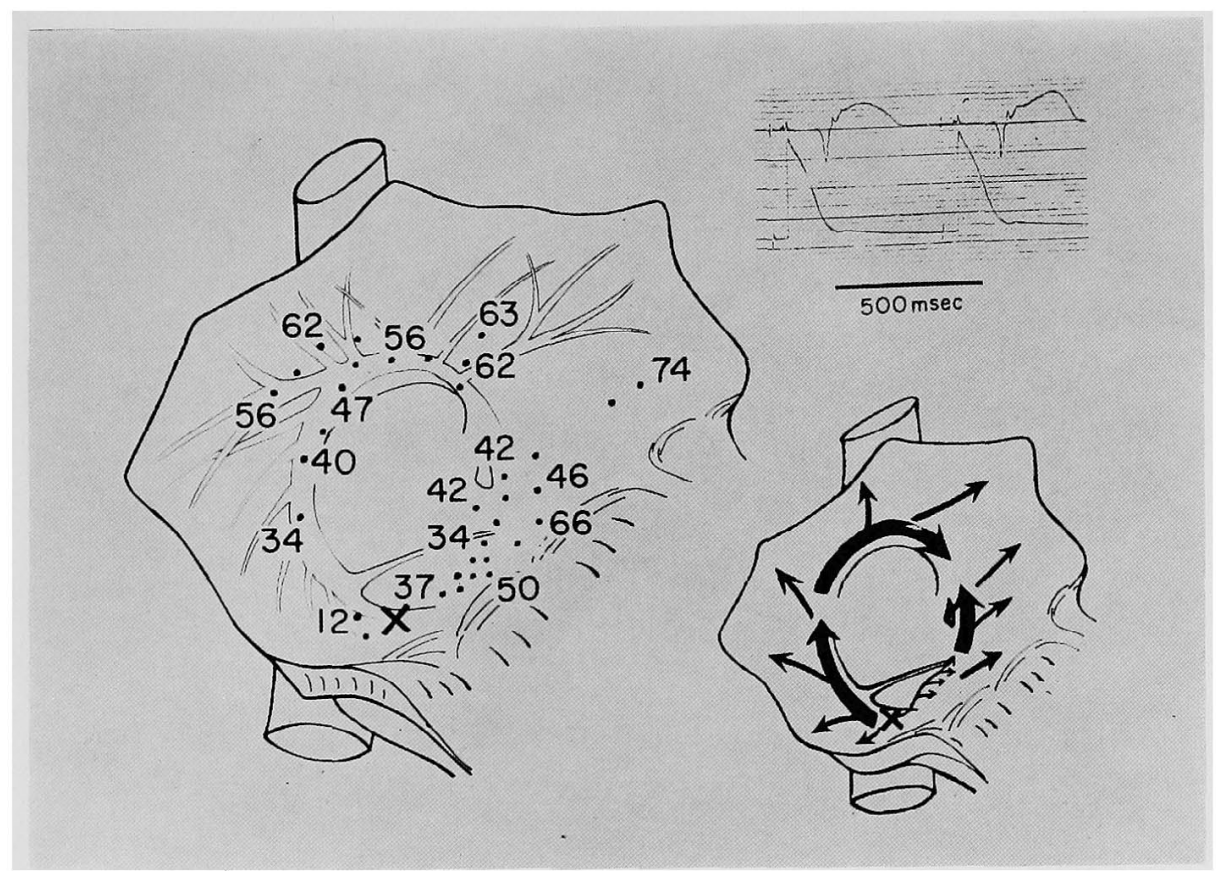

Fig. 6. Activation time of the right atrial endocardium during pacing from RGT. Demonstration of the panels is the same as in Fig. 4.

crista terminalis. On the other hand, the activation wave which was transmitted slowly across the coronary sinus ostium was conducted upward along the septal segment of the crista terminalis. These 2 waves collided at the middle to the upper region of the interatrial septum in $60 \mathrm{msec}$ from the pacing stimulus. So, most of the right atrium was activated by the impulse which was propagated from the activation wave conducted upward along the right segment of the crista terminalis.

When the specimen was paced from the middle portion of the coronary sinus ostium (Fig. 7), the impulse was propagated radially and towards the AV node, and the lower ends of the right and septal segments of the crista terminalis. Two activation waves spread upward respectively along the right and septal segments of the crista terminalis, and collided with each other in 40 to $45 \mathrm{msec}$ at the anterior edge of the superior vena cava orifice. Most of the right atrial muscles was activated by the impulse spread out from the both segments of the crista terminalis.

When the specimen was paced from the left atrial appendage (LAA, Fig. 8), the first region to be activated in the right atrium was located over the region at which the right atrium was connected with Bachmann's bundle, and the region near the SA node was activated early. From these regions, 


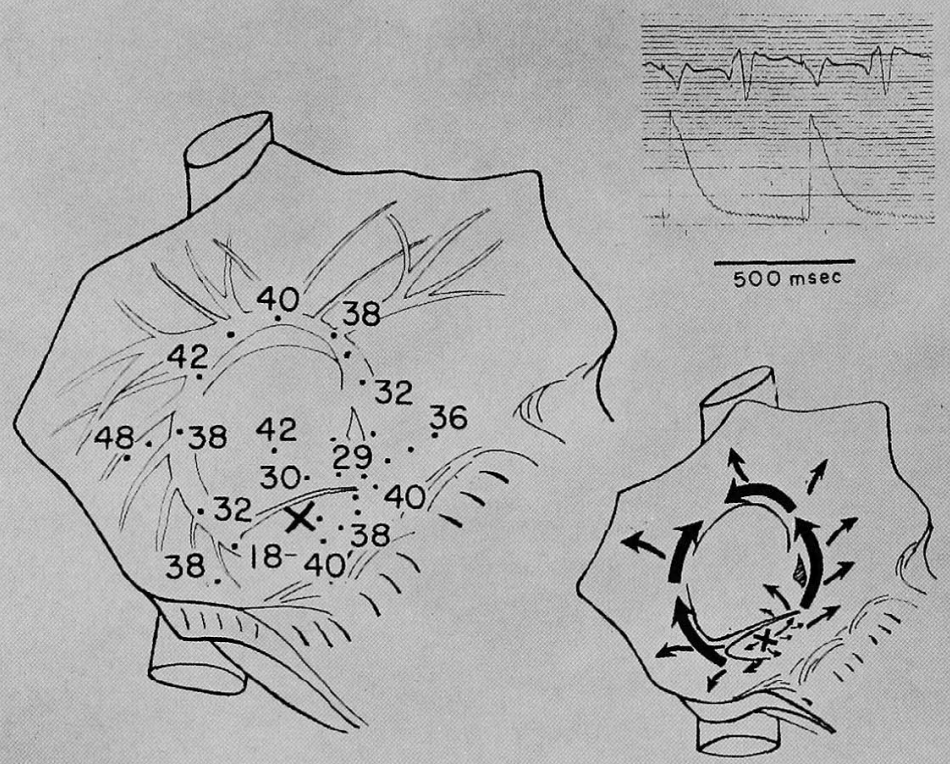

Fig. 7. Activation time of the right atrial endocardium during pacing from mid. GSO. Demonstration of the panels is the same as in Fig. 4.

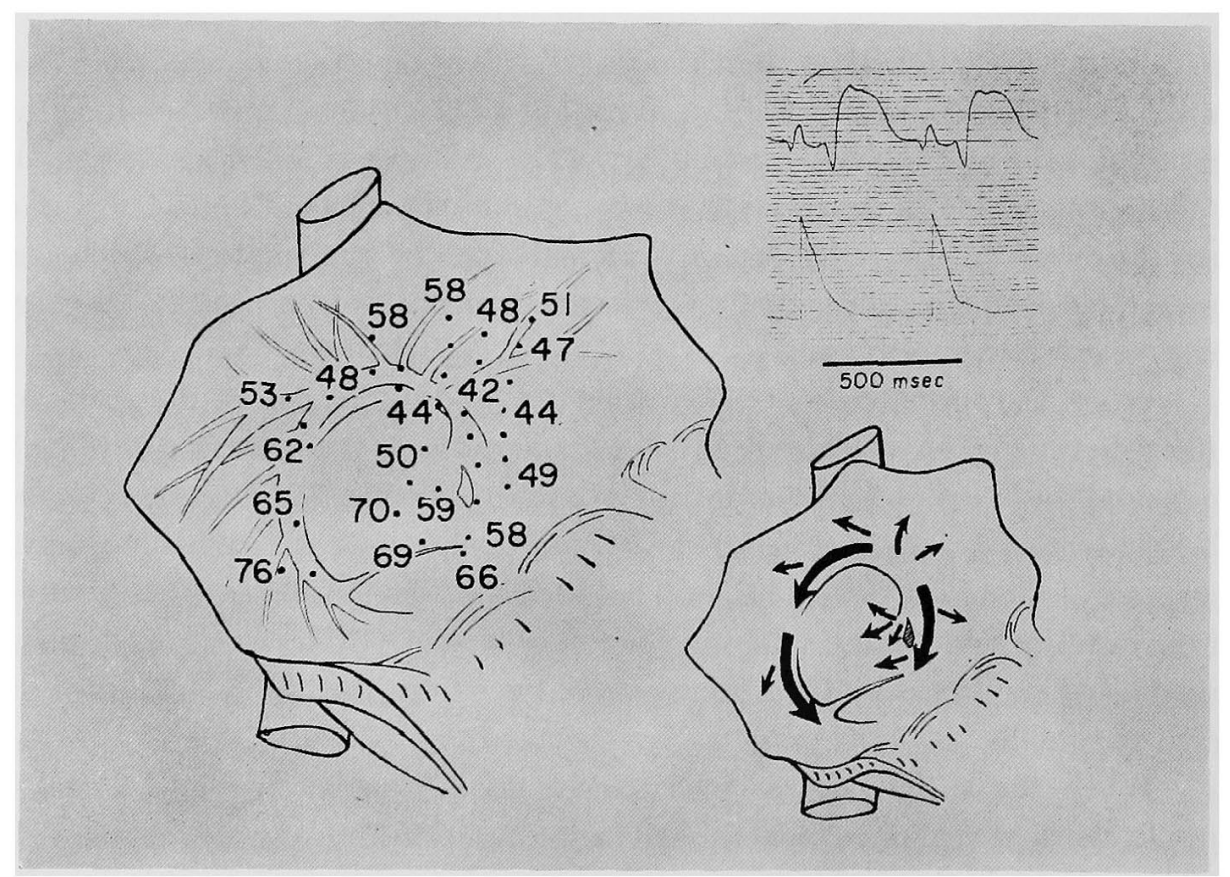

Fig. 8. Activation time of the right atrial endocardium during pacing from LAA. Demonstration of the panels is the same as in Fig. 4. 


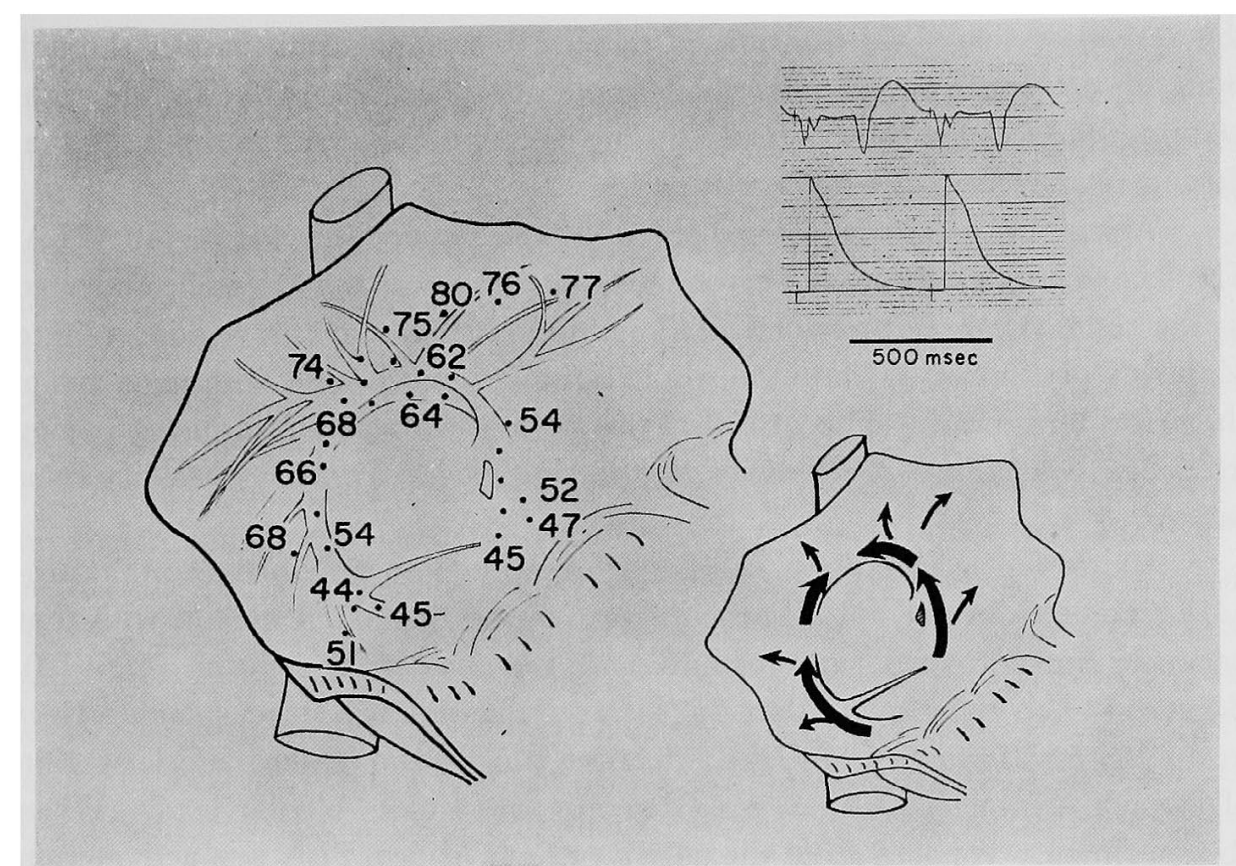

Fig. 9. Activation time of the right atrial endocardium during pacing from PLA. Demonstration of the panels is the same as in Fig. 4.

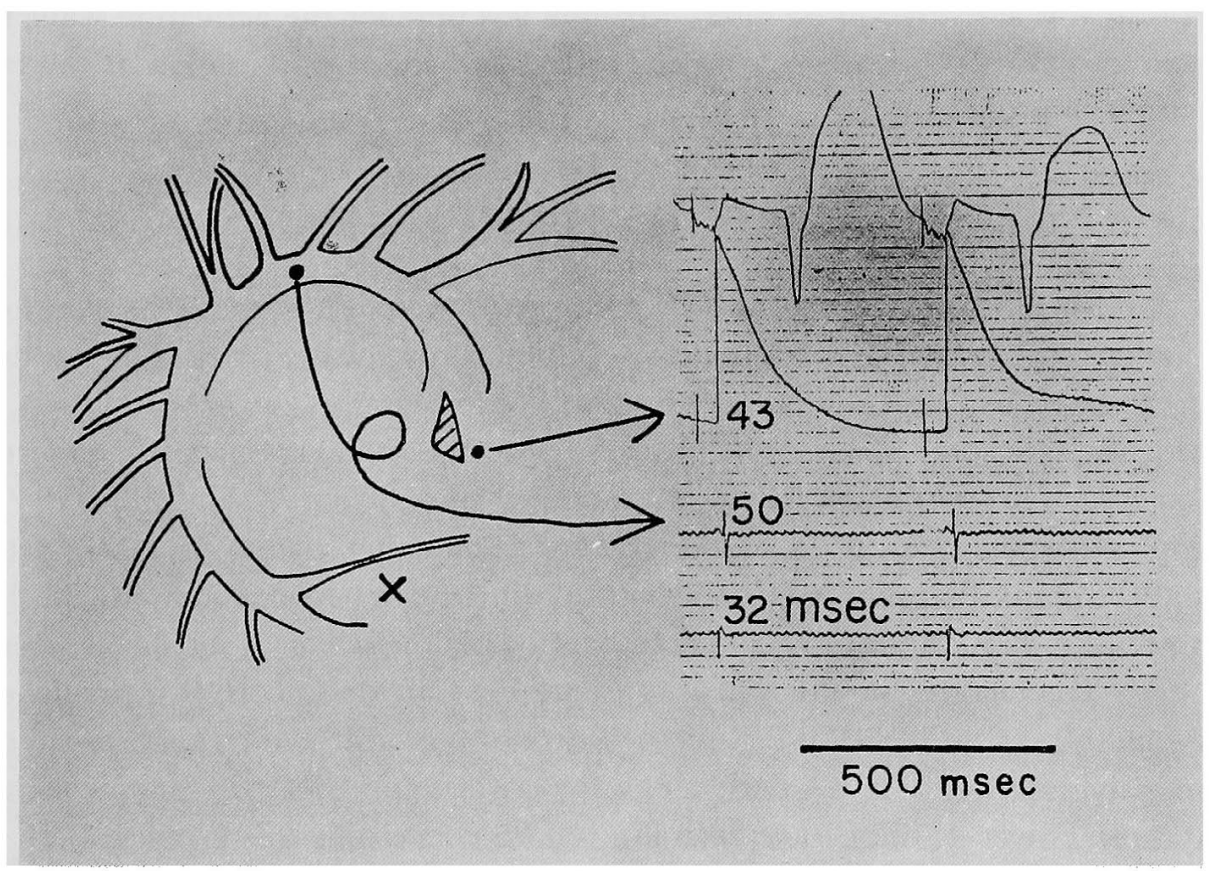

Fig. 10. Activation time during pacing from mid. CSO. Lower 2 electrograms are the close bipolar electrograms from the right atrium (indicated by a dot) and PLA. The posterior region of the left atrium was activated earlier than the middle portion of the interatrial septum. 
the activation wave was transmitted to the other right atrial muscles through both of the segments of the crista terminalis. Consequently, in the right atrium, the spread of the excitation during pacing from LAA was similar to that during pacing from the SA nodal region.

When the specimen was paced from the postero-inferior region (PLA, Fig. 9), the region around the coronary sinus orifice was the first to be activated in the right atrial endocardium. The impulse arrived at this region in 40 to $45 \mathrm{msec}$. It spread out into the whole right atrium through both segments of the crista terminalis. The spread of the right atrial activation during pacing from PLA was almost the same as that during pacing from the coronary sinus ostium.

An attempt was made to compare the conduction time between the coronary sinus ostium and the postero-inferior region of the left atrium with that between the coronary sinus ostium and the interatrial septum (Fig. 10). Because of the experimental set-up, intracellular microelectrodes couldn't get to the left atrial muscle. So, the activation time at the postero-inferior region of the left atrium (PLA) was recorded through a close bipolar lead. When the specimen was paced from the coronary sinus orifice, PLA was activated in $32 \mathrm{msec}$ from the pacing stimulus, and the middle region of the interatrial septum was activated in $43 \mathrm{msec}$. It took less time for the activation wave to conduct between the coronary sinus orifice and the middle region of the interatrial septum.

\section{Discussion}

For a long time, there has been much controversy concerning the question whether specialized or preferential conduction pathways participate in the spread of atrial activation in sinus rhythm. According to Lewis ${ }^{1)}$ and others, ${ }^{2}$ ) the activation wave travels from the sinus node in a radial manner at a uniform velocity. On the other hand, Rothberger and Scherf, ${ }^{13}$ and others ${ }^{51-11)}$ proposed some specialized or preferential conduction pathways. Merideth et al $^{14)}$ did not find any striking structural differences between individual cells composing 3 internodal tracts and ordinary atrial cells except for direct fiberto-fiber connections in the 3 tracts. Paes de Calvalho et al, ${ }^{15}$ ) Yamada et

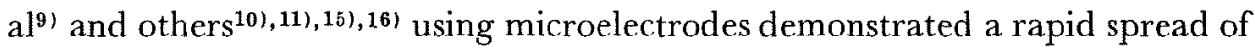
the activation from the SA node along the crista terminalis. In ectopic atrial rhythms, however, there have been few reports concerning this problem. In a study on the excitation sequence of the atrial septum during antegrade atrial and retrograde His bundle pacing, Spach et al ${ }^{17}$ ) found no functional evidence for narrow, specialized internodal tracts except for simultaneous, multiple 
wave fronts. On canine atria, Goodman and coworkers ${ }^{12)}$ observed preferential conduction along the crista terminalis during pacing near the coronary sinus and pacing at the auricular appendage as well as during pacing at the SA node. In the present study, right atrial activation originating from ectopic sites was transmitted faster along the crista terminalis and slower across the coronary sinus ostium than through the ordinary atrial musculature. The approximate conduction velocity was $40-100 \mathrm{~cm} / \mathrm{sec}$ along the crista terminalis, $50 \mathrm{~cm} / \mathrm{sec}$ through the ordinary atrial muscle, and $25 \mathrm{~cm} / \mathrm{sec}$ across the coronary sinus ostium.

In the transitional region from the coronary sinus ostium to the AV node, neuroganglionic cells were reported to exist, ${ }^{18)}$ and Truex and Smythe ${ }^{19)}$ successfully traced tracts of small AV nodal fibers to the coronary sinus region. The AV nodal action potentials are known to display a low rate of depolarization, low (less negative) resting potential, low action potential and poor spike et al in comparison to the atrial action potentials.9 $\left.{ }^{9}, 10\right)$ In the present experiment, in fact, the atrial action potentials, the AV nodal action potentials and the transitional types of these 2 action potentials were obtained from the region of the coronary sinus ostium. The low conduction velocity across the coronary sinus ostium might be explained by these complexed compositions.

From the view point of the conduction between both atria, there have been several studies ${ }^{201-22)}$ dealing with Bachmann's bundle. Bachmann ${ }^{23)}$ in 1916 first called attention to the interatrial band as a structure with a special function in interatrial conduction, and by clamping or ligating this band, he observed interatrial delay or block. Horiba, ${ }^{20}$ ) Wagner et $\mathrm{al}^{21)}$ and Oishi ${ }^{22}$ reported accelerated conduction along the interatrial band. In the present paper, when the specimen was paced from the left atrial appendage, the first place to be depolarized in the right atrium was the region around the superior vena cava orifice and the region covering the site at which Bachmann's bundle was connected with the right atrium. Consequently, the region near the SA node was depolarized relatively early and from these regions, most of the right atrial musculature was activated in a sequence similar to that during sinus rhythm. In this case, the polarity of the atrial deflection in the distant bipolar lead was negative-positive and the initial negative deflection was thought, from determination of activation time, to be caused by the activation wave propagating through the left atrium. If the interatrial band does not display accelerated conduction, the first region to be activated in the right atrium should not be localized in the region above mentioned. It is suggested that this bundle has a function as a preferential conduction pathway for the activation wave not only in the direction from the right atrium to the left one but also in the opposite direction. 
During pacing from the postero-inferior region of the left atrium, the first region to be activated in the right atrium was around the coronary sinus ostium. The impulse was then transmitted to all other parts of the right atrium upward through both segments of the crista terminalis. The propagation process of the right atrial activation became nearly the same as that during pacing from the coronary sinus ostium and in the opposite sequence to that during sinus rhythm.

Except for Bachmann's bundle, there has been no definite concept concerning the interatrial conduction pathway. According to Oosawa, ${ }^{24}$ the right and left atrial musculature are separated from each other in most portions of the interatrial septum and the activation generated in the SA node is transmitted to the left atrial muscle in the upper portion of the interatrial septum through the systema septi sinistra. Oishi, ${ }^{22)}$ Spach et al, ${ }^{25)}$ and Matsuoka ${ }^{26)}$ observed an activation wave which travels from right to left or in the opposite direction in the postero-inferior region of the atria. Matsuoka reported that when Bachmann's bundle was ligated or cauterized, the activation wave originating in the left atrial appendage seemed to be transmitted to the right atrium through the postero-inferior region of the left and right atria, and then spread into the anterior right atrium. Recently, Scherlag et al ${ }^{27}$ studied histologically and electrophysiologically the Ligament of Marshall, which is a vestigial tissue encompassing portions of the embryonic sinus venosus and left cardinal vein. They suggested the existence of an interatrial pathway connecting the posterior portions of the right atrium with the inferior-posterior left atrium along the coronary sinus. The findings in the present experiments are in agreement with these authors and suggest the existence of a preferential pathway along the coronary sinus vein.

During pacing from the right side of the lower interatrial septum (LIS), the atrial deflection in the distant bipolar electrogram was positive in most cases. This fact may be explained in part by the relatively large part of the activation wave which was directed downward, although the stimulating point was located at the lower end of the right atrium. In the interatrial septum, the impulse which was originated from LIS might be conducted to the left atrium through the upper portion of the septum as above discussed. The left atrium might be activated downward from the upper portion of it, and the positive atrial deflection during pacing from LIS might be attributed partially to the downward activation wave in the left atrium.

During pacing from the coronary sinus ostium, the atrial deflection in the distant bipolar electrogram displayed a negative complex and in this case the spread of the right atrial activation was directed upward.

Borrman and Meek $^{28}$ observed in the study of dogs that after the SA node 
was destroyed by implanting radon, the initiation of the impulse occurred in the coronary sinus region and the coronary sinus rhythm was electrocardiographically indistinguishable from sinus rhythm. Moore et $\mathrm{al}^{31}$ and others ${ }^{4}$ concluded that the $\mathrm{P}$ waves in leads II, III and $\mathrm{aV}_{\mathrm{F}}$ were variable or did not necessarily inverted during coronary sinus stimulation and stimulation of the lower interatrial septum. On the other hand, Lancaster and others ${ }^{29}$ observed that the $P$ waves in leads II, III and $a V_{F}$ were negative in most cases during coronary sinus rhythm elicited by stimulation through an electrode catheter. Lau ${ }^{30}$ suggested that one of the major factors causing this discrepancy was the pacing site which had been used by these investigators. That is, when the stimulating site was within the coronary sinus, the $\mathbf{P}$ wave was negative though the $P$ wave was not inverted when the inside of the coronary sinus was avoided. Although the results obtained in the present experiments were in agreement with the notion of Lau, it seems to be more reasonable that the difference in conduction velocity between along the crista terminalis and across the coronary sinus ostium is a major factor.

In the left atrial rhythms, according to Harris, ${ }^{31)} \mathbf{P}$ waves displayed negative deflections during stimulation at the posteroinferior region of the left atrium and in the frontal plane they were indistinguishable from those during coronary sinus rhythm. Lau obtained similar results. In the present study, the results confirmed their findings. The fact that $\mathbf{P}$ waves during coronary sinus rhythm are similar in the frontal plane to those during inferior left atrial rhythm can be explained by postulating a preferential tract along the coronary sinus vein between the postero-inferior portions of the both atria. And this thesis was supported by the present study of activation sequence.

Atrio-ventricular conduction time, corresponding to the P-R interval of electrocardiogram, was determined as described above. During pacing from the selected sites on the crista terminalis, A-V conduction time was shorter than that during pacing from sinus region. It was also shorter during pacing from the coronary sinus ostium. It has been mentioned that the P-R interval is slightly short in coronary sinus rhythm in comparison to spontaneous sinus rhythm in the same patient. ${ }^{32}$ ) These facts may be explained by the shorter anatomic distance from the site of origin of ectopic rhythm to the AV node.

In clinical electrocardiography, coronary nodal rhythm is diagnosed when the $P-R$ interval is between 0.02 and $0.10 \mathrm{sec}$ and the $P$ waves in leads $I$ and II are upright. ${ }^{33)}$ The reason normal $P$ waves may be expected in coronary nodal rhythm is explained by the concept that the site of origin of this rhythm may be located at the region closest to the tail of the SA node. ${ }^{33}$ ) Although LIS did not seem to coincide with this site, the upward atrial deflection and the shorter AV interval during pacing from LIS accorded with the 
features of this rhythm. Therefore, the origin of coronary nodal rhythm remains to be further studied.

\section{ACKNOWLEDGEMENTS}

The author gratefully thanks Professor Kazuo Yamada, Professor Mitsuharu Okajima, and Associate Professor Junji Toyama for their valuable suggestions and supports. The author is also grateful to Dr. Kay Millar, Associate Professor of Medicine, University of Utah Medical Center, for her kindness in correcting the English in the manuscript, and Dr. Katashi Ohkuma for his help and advice.

\section{REFERENCES}

1. Lewis T: The mechanism and graphic registration of the heart beat. Shaw \& Sons, London, 1920

2. Pucch P, Esclavissat MM, Sodi-Pallares D, Cisneros F: Normal auricular activation in dog's heart. Am Heart J 47: 174, 1954

3. Moore EN, Jomain SL, Stuckey JH, Buchanan JW, Hoffman BF: Studies on ectopic atrial rhythms in dogs. Am J Gardiol 19: 676, 1967

4. Waldo AL, Vitikainen KJ, Kaiser GA, Malm JR, Hoffman BF : The P wave and P-R interval. Effects of the sites of origin of atrial depolarization. Circulation 42:653,1970

5. Eyster JAE, Meek WJ: Experiments on the origin and propagation of the impulse in the heart. Heart 5: 119-135, 1913-1914

6. Takayasu M: Studies on the electrocardiogram recorded with the cathode ray oscillograph. Jap Circulat J 3: 293-298, 381-384, 447-450, 1937-1938

7. James $\mathrm{TN}$ : The connecting pathways between the sinus node and $\mathrm{A}-\mathrm{V}$ node and between the right and left atrium in the human heart. Am Heart J 66: 498, 1963

8. James TN: Anatomy of the cardiac conduction system in the rabbit. Circulat Res 20: 638,1967

9. Yamada K, Horiba M, Sakaida $Y$, Okajima M, Horibe H, Muraki H, Kobayashi T, Miyauchi A, Oishi $\mathrm{H}$, Nonogawa A, Ishikawa $\mathrm{K}$, Toyama $\mathrm{J}$ : Origination and transmission of impulse in the right auricle. Jap Heart J 6: 71, 1965

10. Matsuda K, Hoshi T, Kameyama S: Action potential of the atrioventricular node (Tawara). Tohoku J exp Med 68: 8, 1958

11. Sano T, Yamagishi S: Spread of excitation from the sinus node. Circulat Res 16: 423, 1965

12. Goodman D, van der Steen ABM, van Dam RT: Endocardial and epicardial activation pathways of the canine right atrium. Am J Physiol 220: 1, 1971

13. Rothberger CJ, Scherf D: Zur Kenntnis der Erregungs-ausbreitung vom Sinusknoten auf den Vorhof. Z Gesamte Exp Med 53: 792, 1926

14. Merideth J, Titus JL: The anatomic atrial connections between sinus and A-V node. Circulation 37: 566, 1968

15. Paes de Carvalho A, de Mello WC, Hoffman BF: Electrophysiological evidence for specialized fiber types in rabbit atrium. Am J Physiol 196: 483, 1959

16. Horibe $\mathrm{H}$ : Studies on the spread of the right atrial activation by means of intracellular microelectrode. Jap Circulat J 25: 583, 1961

17. Spach MS, Lieberman M, Scott JG, Barr RC, Johnson EA, Kootsey JM: Excitation sequences of the atrial septum and the AV node in isolated hearts of the dog and rabbit. Circulat Res 29: 156, 1971 
18. Kung SK, Mobitz W: Herzblockstudien 1. Teil: Ueber die normale Histologie des Reizleitungssystems und pathologisch-histologische Befunde an blockierten Herzen des Menschen. Arch exp Pathol Pharmak 155: 295, 1930

19. Truex RC, Smythe MO: Recent observation on the human cardiac conduction system, with special considerations of the atrioventricular node and bundle. Electrophysiology of the Heart, ed by Taccardi B, Marchetti G, New York, Pergamon Press, 177-198, 1965

20. Horiba M: Stimulus conduction in atria studied by means of intracellular microelectrode. Part I. That in Bachmann's bundle. Jap Heart J 4: 333, 1963

21. Wagner WL, Lazzara $R$, Weiss RM, Hoffman BF: Specialized conducting fibers in the interatrial band. Circulat Res 18: 502, 1966

22. Oishi $\mathrm{H}$ : Manner of stimulus conduction in atria. Jap Heart J 8: 276, 1967

23. Bachmann G: The inter-auricular time interval. Am J Physiol 41: 309, 1916

24. Oosawa $\mathrm{M}$ : Histological study on the cardiac conduction system in the atrial portion of the dog's heart. Jap Circulat J 23: 548, 718, 1014, 1178, 1959

25. Spach MS, King TD, Barr RC, Boaz DE, Morrow MN, Herman-Giddens S: Electrical potential distribution surroundung the atria during depolarization and repolarization in the dog. Circulat Res 24: 857, 1969

26. Matsuoka S: Experimental studies on the auricular waves. Jap Girculat J 21: 1, 1957

27. Scherlag BJ, Yeh BK, Robinson MJ: Inferior interatrial pathway in the dog. Circulat Res 31: 18, 1972

28. Borrman MC, Meek WJ: Coronary sinus rhythm. Rhythm subsequent to destruction by radon of the sino-auricular nodes in dogs. Arch Intern Med 47:957, 1931

29. Lancaster JF, Leonard JJ, Leon DF, Kroets FW, Shaver JA: The experimental production of coronary sinus rhythm in man. Am Heart J 70:89, 1965

30. Lau SH, Gohen SI, Stein E, Haft JI, Rosen KM, Damato AN: P waves and P loops in coronary sinus and left atrial rhythm. Am Heart J 79: 201, 1970

31. Harris BC, Shaver JA, Gray S, Kroets FW, Jeonard JJ : Left atrial rhythm. Experimental production in man. Circulation 37: 1000, 1968

32. Scherf D, Cohen J: The atrioventricular node and selected cardiac arrhythmias. Grune and Stratton, New York, 1964

33. Katz LN, Pick A: Clinical Electrocardiography. I. The arrhythmias. Lea and Febiger, Philadelphia, 1956 